\title{
MDR1 deficiency impairs mitochondrial homeostasis and promotes intestinal inflammation
}

\author{
G-T Ho ${ }^{1,2}$, RE Aird ${ }^{1,3}$, B Liu ${ }^{4}$, RK Boyapati ${ }^{1,2}$, NA Kennedy ${ }^{2}$, DA Dorward ${ }^{1}$, CL Noble ${ }^{2}$, T Shimizu ${ }^{5}$, \\ RN Carter ${ }^{3}$, ETS Chew ${ }^{1}$, NM Morton ${ }^{3}$, AG Rossi ${ }^{1}$, RB Sartor ${ }^{4}$, JP Iredale ${ }^{1,6}$ and J Satsangi ${ }^{2}$
}

The multidrug resistance-1 (MDR1) gene encodes an ATP-dependent efflux transporter that is highly expressed in the colon. In mice, loss of MDR1 function results in colitis with similarities to human inflammatory bowel diseases (IBD). Here, we show that MDR1 has an unexpected protective role for the mitochondria where MDR1 deficiency results in mitochondrial dysfunction with increased mitochondrial reactive oxygen species (mROS) driving the development of colitis. Exogenous induction of mROS accelerates, while inhibition attenuates colitis in vivo; these effects are amplified in MDR1 deficiency. In human IBD, MDR1 is negatively correlated to SOD2 gene expression required for mROS detoxification. To provide direct evidential support, we deleted intestinal SOD2 gene in mice and showed an increased susceptibility to colitis. We exploited the genome-wide association data sets and found many ( $~ 5 \%)$ of IBD susceptibility genes with direct roles in regulating mitochondria homeostasis. As MDR1 primarily protects against xenotoxins via its efflux function, our findings implicate a distinct mitochondrial toxin + genetic susceptibility interaction leading to mitochondrial dysfunction, a novel pathogenic mechanism that could offer many new therapeutic opportunities for IBD.

\section{INTRODUCTION}

Inflammatory bowel diseases (IBD) are complex immunemediated conditions that can be subclassified into the clinically distinct entities of Crohn's disease (CD) and ulcerative colitis (UC). ${ }^{1,2}$ Both are debilitating relapsing inflammatory conditions, which affect an estimated 4 million people in the USA and Europe with increasing prevalence in the developing world. $^{3} \mathrm{CD}$ is characterized by discontinuous aphthous mucosal ulcerations and inflammation occurring most commonly in the distal small intestine (ileum) and variably across the intestinal tract. ${ }^{2} \mathrm{UC}$ affects only the large bowel (colon) in a confluent manner and is characterized by superficial mucosal inflammation. ${ }^{4}$ Increasingly evident both in clinical and molecular studies is the concept that IBD involves multiple distinct and also, common pathogenic mechanisms. The powerful genome-wide association studies implicate more than 200 genetic susceptibility loci including genes in biological pathways regulating innate (e.g., NOD2 and autophagy) and adaptive (e.g., Th17, MHC) immune responses, cytokine production, lymphocyte activation, and bacterial response.,

One major mechanistic theme that is shared between UC and $\mathrm{CD}$ is the role of intestinal epithelial dysfunction and injury. ${ }^{7}$ The intestinal epithelium is uniquely in contact with high concentrations of bacteria with their metabolic products, immune-active ligands, damage-associated molecular products (DAMPs), xenobiotics, and environmental toxins. ${ }^{8}$ Thus, a central component of IBD pathogenesis is the deregulation of protective mechanisms that maintain cellular homeostasis and resultant epithelial dysfunction. Multidrug resistance-1 (MDR1) is a key mediator of one such mechanism ${ }^{9}$ by actively extruding more than 4,000 diverse products, including xenotoxins, out of the intracellular environment. ${ }^{10}$ MDR1 belongs to the ATP-binding cassette, ABC-transporter family of transmembrane transporters. These proteins bind ATP and use the energy to drive the transport of various molecules across all cell membranes. Genetic variations in this family of genes

${ }^{1}$ MRC Centre for Inflammation Research, Queens Medical Research Institute, University of Edinburgh, Edinburgh, UK. ${ }^{2}$ Gastrointestinal Unit, Western General Hospital, University of Edinburgh, Edinburgh, UK. ${ }^{3}$ University/BHF Centre for Cardiovascular Science, University of Edinburgh, Edinburgh, UK. ${ }^{4}$ Departments of Medicine, Microbiology and Immunology, Center for Gastrointestinal Biology and Disease, University of North Carolina, Chapel Hill, North Carolina, USA. ${ }^{5}$ Department of Advanced Aging Medicine, University of Chiba, Chiba, Japan and ${ }^{6}$ University of Bristol, Bristol, UK. Correspondence: G-T Ho (gho@ed.ac.uk) 
contribute to several human disorders including cystic fibrosis, neurological diseases, retinal degeneration, anemia, cholesterol, and bile transport defects. ${ }^{9}$

Epithelial cells of the distal intestinal tract have very high MDR1 expression reflecting the importance of its cytoprotective role at the gut luminal interface. ${ }^{11}$ In the mouse, the $m d r 1 a$ gene encodes PgP170. Constitutive genetic deletion of this gene results in chronic spontaneous colitis as the mutants age with $\sim 50 \%$ penetrance when $>6$ months of age. ${ }^{12}$ Interestingly, the mdr1a-deficient mice display no other spontaneous clinical phenotype, underlining the dominant physiological role of mdrla in the gut. Its importance in colonocytes is further highlighted by the lack of rescue from colitis when $m d r 1 a$-deficient mouse were reconstituted with $m d r 1 a^{+}$bone marrow stem cells. ${ }^{12}$ In human IBD, several studies have shown a downregulation of intestinal MDR1 expression. ${ }^{13,14}$ MDR1 has been linked to IBD in early human candidate genetic studies with disease-associated variants associated with low MDR1 expression. ${ }^{15}$ These findings implicate the loss of MDR1 function as a contributory factor in the pathogenesis of IBD.

Although an underlying increase in intestinal permeability is considered as the dominant factor for the development of $m d r 1 a$-deficient colitis, the preceding steps leading to this late stage are not fully clarified. ${ }^{16}$ In this study, we show for the first time that MDR1 confers a protective role to the gut epithelial mitochondria and that MDR1 deficiency results in mitochondrial dysfunction driving the development of colitis. The mitochondria play a key role in many physiological processes such as energy production, regulation of cell death, and immune response. Given that MDR1 is critically positioned at the interface between mitochondria and the luminal environment, this protective mechanism opens up a fresh perspective in how deregulation of mitochondria homeostasis influences the development of colitis.

\section{RESULTS}

Mdr1a-deficient colonic epithelium shows an accumulation of damaged mitochondria

Using transmission electron microscopy, we showed that the mdrla-deficient mice displayed a preferential accumulation of damaged and degenerating mitochondria in colonic epithelial cells (CECs) in the inflamed colon (Figure 1a). CECs harboring these mitochondrial abnormalities were found in higher frequencies in mdrla-deficient mice compared to noninflamed colon, ileum, and liver in wild-type (WT) mice $(14 \%$ vs. $<2 \%$ overall frequency) (Figure $\mathbf{1 b}$; Supplementary Figure S1a online). These features were not present in il10-deficient mice CECs, a spontaneous chronic colitis control group with a defined inflammatory mechanism ${ }^{17}$ (Supplementary Figure S1b) and only in necrotic CECs during acute dextran-sulfate sodium colitis (DSS). Immunohistochemistry analysis of mdrla-deficient colons revealed increased colonic epithelial p62, PTEN-induced kinase 1, and superoxide dismutase-2 (SOD2) staining (Figure 1c). p62 is a ubiquitin-binding protein that promotes autophagic degradation of protein aggregates, recognizes damaged mitochondria, and recruits them to the isolation membrane through its interaction with LC3. ${ }^{18}$ PTEN-induced kinase 1 is involved in specific autophagic clearance of damaged mitochondria (or mitophagy); ${ }^{19}$ whereas SOD2 plays a role in detoxifying mitochondrial reactive oxygen species (ROS). Furthermore, isolated CECs confirmed higher p62, increased microtubule-associated protein 1 light chain $3-\beta$, LC3-I to phosphatidylethanolamine-conjugated or LC3-II ratio indicative of heightened autophagy; and parkin expression in mdrla deficiency (Supplementary Figure S2a). Parkin translocates to mitochondria upon dissipation of mitochondria membrane potential and cooperates with PTEN-induced kinase 1 during mitophagy. CEC voltage-dependent anion channel expression and mitochondria DNA were similar to $\mathrm{WT}$, suggesting that these changes were not due to changes in mitochondria content (Figure 1c, Supplementary Figure S2b). Mitochondria isolated from mdrla-deficient CECs were more sensitive to mitochondrial membrane damage by carbonyl cyanide m-chlorophenyl hyrdrazone (CCCP), a known damaging agent (Figure 1d). Furthermore, we detected increased circulating plasma mitochondrial DNA, a known inflammatory DAMP in $m d r 1 a$-deficient mice with colitis (Figure 1e). The mdr1a-deficient colons have increased epithelial cell turnover and death, respectively (Supplementary Figure S2c and d). MDR1-deficient mice exhibited clear evidence of colonic epithelial mitochondrial damage with increased epithelial dysfunction and cell death.

\section{MDR1 deficiency results in mitochondria dysfunction}

To study the functional consequence of MDR1 deficiency on the mitochondria, we investigated the cellular energetics of primary colonic explants of $m d r l a^{-/-}$and WT mice. We found lower basal $\mathrm{O}_{2}$ consumption rate in $m d r 1 a^{-1-}$ colon consistent with impaired mitochondrial function and energy production (Figure 2a). We further knocked down MDR1 expression in vitro by transducing short hairpin MDR1 (shMDR1) in the human T84 CEC cell line (Supplementary Figure S3a). Although we found no differences in $\mathrm{O}_{2}$ consumption rate (Supplementary Figure S3b), MDR1 deficiency in T84 CECs resulted in lower spare respiratory capacity (Figure $\mathbf{2 b}$ ). Spare respiratory capacity is the extra mitochondrial capacity available in a cell to produce energy under conditions of increased work and stress and is thought to be important for long-term cell survival and function. ${ }^{20}$ Dysfunctional or damaged mitochondria produce increased levels of ROS and the mitochondria are the major source of ROS for most cells. ${ }^{21}$ In line with our observations in mdrladeficient mouse CECs, we found increased levels of mitochondrial ROS (mROS) in shMDR1 T84 CECs at baseline (Figure 2c); and following artificial induction with rotenone and antimycin by fluorescence of MitoSOX (a mitochondrial superoxide indicator) (Figure 2d). Furthermore, we showed that MDR1 knockdown resulted in higher p62 and SOD2 protein expressions following rotenone treatment in shMDR1 CECs (Supplementary Figure S3c). We transfected 


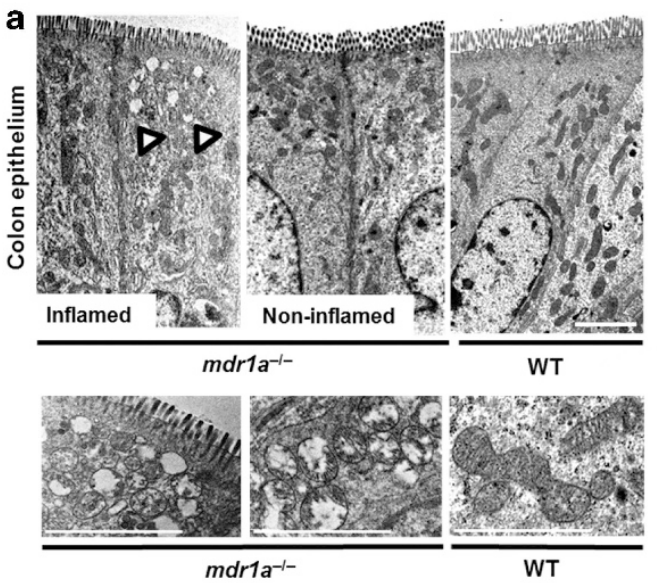

C H\&E

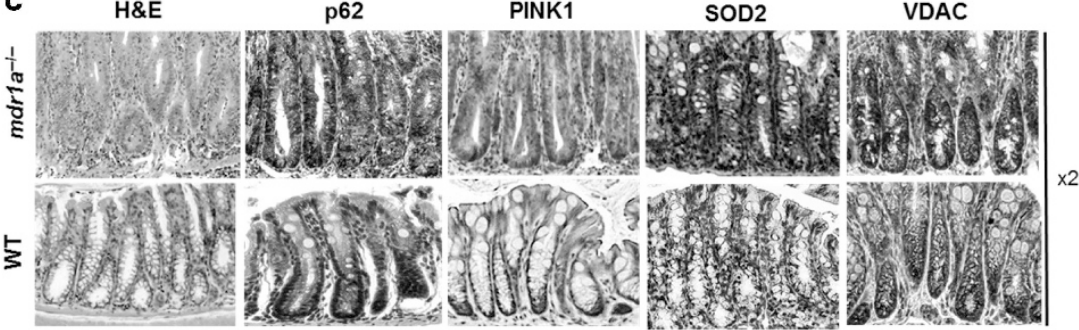

b

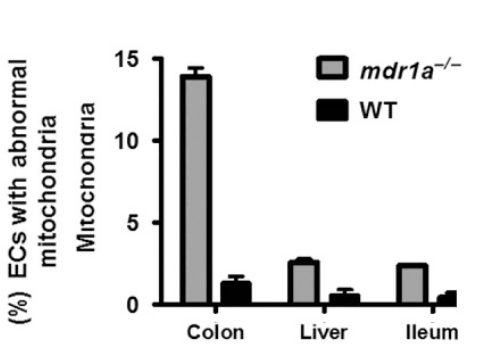

d

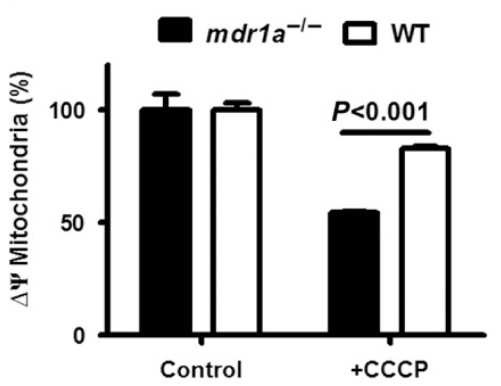

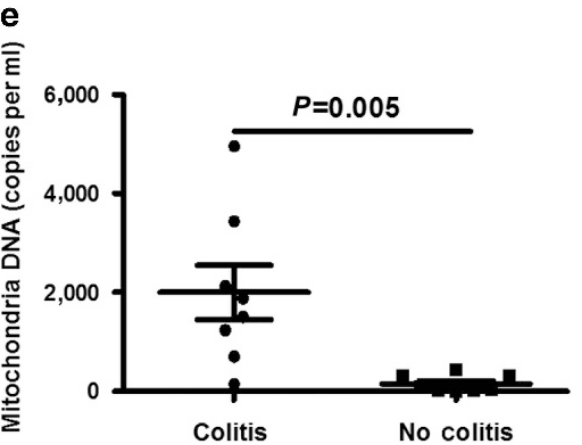

Figure 1 Damaged mitochondria accumulate within mdr1a-deficient colonic epithelium: (a) Representative TEM ( $n=6$ per group) of colonic epithelium. Scale bar $2 \mu \mathrm{m}$. Yellow arrows denoting damaged mitochondria. (b) Quantitative analyses of CEC containing damaged mitochondria ( $n=6$ per group). (c) Representative H\&E and immunohistochemistry of colon (p62, PINK1, SOD2, and VDAC) ( $n=6$ per group). (d) Loss of mitochondria membrane potential, JC-1 fluorescence in isolated mitochondria ( $n=6$ per group) (e) Plasma qPCR of mitochondrial DNA mdr1a ${ }^{-1-}$ mice with colitis vs. no colitis ( $n=8$ per group). All data represent mean \pm s.e.m. CEC, colonic epithelial cells; H\&E, hematoxylin and eosin; mdr1, multidrug resistance-1; PINK1, PTEN-induced kinase 1; qPCR, quantitative PCR; SOD2, superoxide dismutase-2; TEM, transmission electron microscopy; VDAC, voltage-dependent anion channel; WT, wild type. A full color version of this figure is available at the Mucosal Immunology journal online.
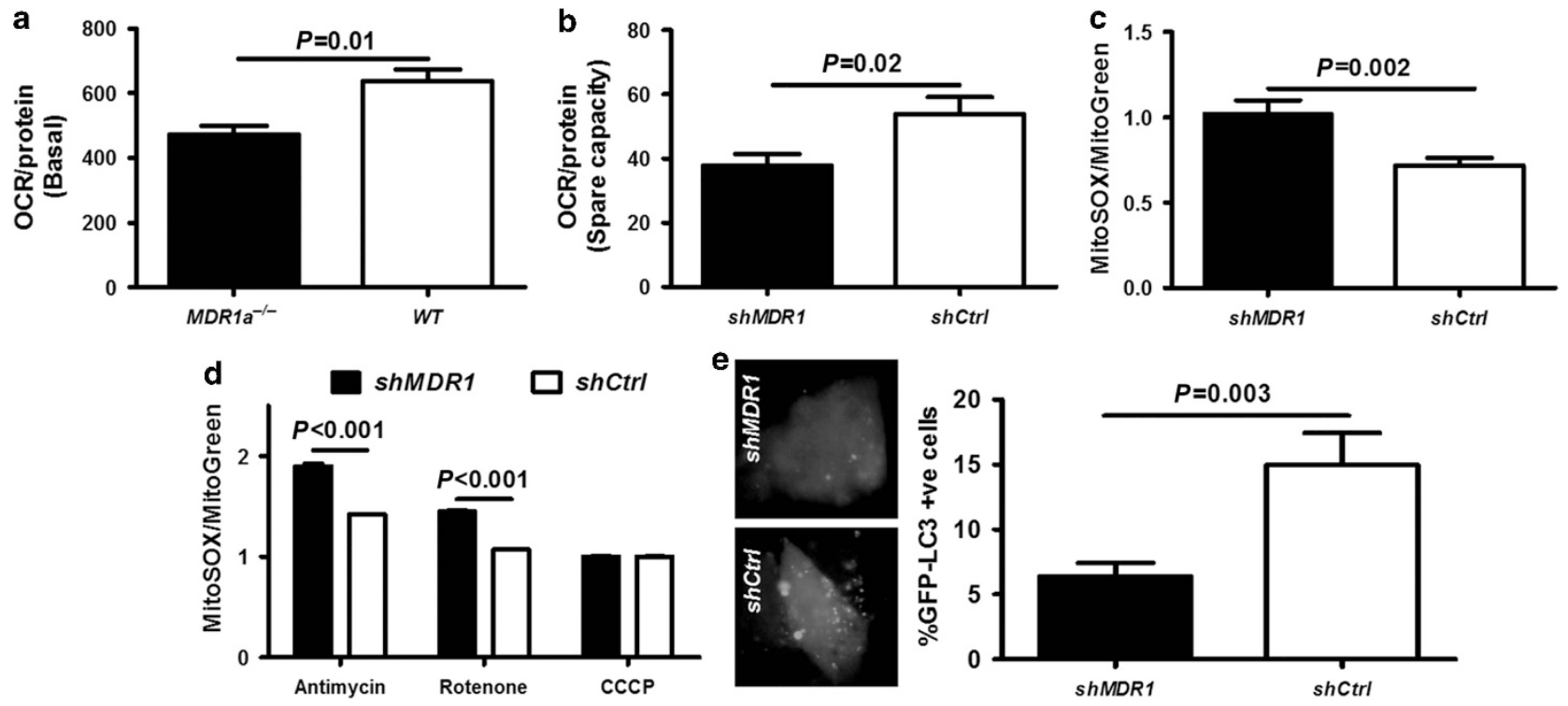

Figure 2 MDR1 deficiency results in mitochondria dysfunction: (a), OCR (pmol $\mathrm{min}^{-1}$ ) in primary colonic explant in mdr1a-deficient vs. WT, respectively $(n=4)$. (b) SRC of T84 shMDRI and shCtrl (five replicates). (c) Normalized MitoSOX/Mitotracker Green fluorescence T84 shMDR1 vs. shCtrl, ( $n=11$ per group, representative of three independent experiments. (d) Relative MitoSOX/Mitotracker Green fluorescence to respective untreated group in T84 shMDR1 vs. shCtrl ( $n=8$ per treatment group, representative of three independent experiments). (e) Quantification of GFP-LC3 shMDR1 vs. shCtrl ( $n=12$ slides per group). \%GFP-LC3 + ve cells per number of cells in 10 fields $(\times 40$ microscopy). All data represent mean \pm s.e.m. MDR1, multidrug resistance-1; OCR, oxygen consumption rate; shMDRI, short hairpin MDRI; SRC, spare respiratory capacity; WT, wild type. A full color version of this figure is available at the Mucosal Immunology journal online. 
LC3-GFP and found reduced autophagy (Figure 2e); and LC3II protein after inhibition of its degradation by bafilomycin (Supplementary Figure S3d) in shMDR1 relative to shCtrl CECs. This suggests a depressed clearance that compounds mitochondria dysfunction. Degenerating mitochondria in CECs are notably present in mice models with primary autophagy (Irgm and Atg16l1), ${ }^{22,23}$ secondary autophagy impairments due to defective ER stress, ${ }^{24}$ and NLRP6 inflammasome activity. ${ }^{25}$ Our data collectively show that loss of MDR1 results in mitochondria dysfunction with impaired bioenergetics, increased mROS production, and perturbed autophagic clearance.

\section{Experimental induction of colonic mitochondria ROS influences the development of colitis in MDR1a deficiency} We then sought to investigate if induction of mROS can accelerate the development of colitis in mdrla deficiency. We developed an in vivo protocol to test this further by directly administrating rotenone with low dose DSS $(0.25 \%)$ into the colon of mdrla-deficient and WT mice. We chose rotenone to specifically focus on the effects of mROS and to test its early effects prior to full induction of DSS colitis. Rotenone is primarily an inhibitor of mitochondrial complex I of electron transport chain, which results in increase mROS production and at higher doses, cellular bioenergetic deficit. ${ }^{26}$ Rotenone is a well-described triggering factor for rodent models of Parkinson's disease (PD) and is administered systemically (by injection) and/or orally. ${ }^{27}$ Given that direct colonic administration in vivo is a new approach, we titrated the colonic rotenone concentration to $100 \mu \mathrm{M}$ (equating to $\sim 1 \mathrm{mg} \mathrm{kg}^{-1}$ ) without systemic ill effects, notably respiratory, neurological and motor deficits (Supplementary Figure S4a). Drug dosing data derived from rodent rotenone-induced PD ranged between 2.5 and $15 \mathrm{mg} \mathrm{kg}^{-1}$ systemically to $0.25-100 \mathrm{mg} \mathrm{kg}^{-1}$ orally, ${ }^{26}$ with increased mortality is observed in the former approach. Within $24 \mathrm{~h}$ of $100 \mu \mathrm{m}$ rotenone administration, we found that this is sufficient to trigger increased leukocyte infiltration and colitis in mdrla-deficient mice, which are not present in WT mice (Supplementary Figure S4b, c) associated with increased CEC death in mdrla-deficient colon (Supplementary Figure S4d, e). We expanded our protocol where we directly administered $500 \mu \mathrm{l}$ of $100 \mu \mathrm{m}$ of rotenone three times per week over 4 weeks in the absence of DSS (Figure 3a). In mdr1a-deficient mice with no prior evidence of colitis, we found that this accelerated the onset of colitis compared to vehicle (Figure $\mathbf{3 b}$ and $\mathbf{c}$ ). In mdrla-deficiency, rotenone-treated mice have more severe colitis compared to the vehicle group (Figure 3d-f). Isolated CECs from rotenonetreated $m d r 1 a$-deficient mice have higher pro-inflammatory il6 and $t n f-\alpha$ gene expressions (Supplementary Figure S4f). In addition to triggering spontaneous colitis, exogenous induction of mucosal mROS using rectal rotenone treatment (Figure 3g) rendered the $m d r l a$-deficient mice more susceptible to acute DSS colitis exhibiting more severe clinical and histological evidence of inflammation (Figure $\mathbf{3} \mathbf{h}-\mathbf{j}$ ). To investigate the effects of rotenone on the epithelial barrier, we further showed that shMDR1 compared to shCtrl CECs have an increased sensitivity to rotenone, CCCP, and cisplatin-induced cell death (Supplementary Figure S5a, b) and displayed an increased loss of barrier function as measured by transepithelial electric resistance following culture with rotenone (Supplementary Figure S5c). In line with our in vivo data, we also showed that mROS induced by rotenone triggered increased colonic epithelial interleukin-8 production induced by flagellin and bacterial CpG (these ligands chosen as our T84 CECs express TLR5 and TLR9) (Supplementary Figure S6d), indicating that mROS triggers CEC death and innate inflammatory responses. Collectively, these data suggest that abnormal mucosal mROS driven by an exogenous trigger can influence the onset and severity of colitis in a genetically susceptible host, in this respect, MDR1 deficiency.

\section{Inhibition of mitochondria ROS effects attenuates and promotes recovery from colitis in vivo}

Next we investigated whether inhibiting the effects of mROS can prevent or attenuate the development of colitis. Mitoquinone (MitoQ) is a mitochondrial specific antioxidant with coenzyme $Q_{10}$ covalently attached to a triphenylphosphonium molecule, which selectively accumulates within the mitochondria. MitoQ protects the mitochondria membrane from mROSinduced lipid peroxidation and acts as a ROS scavenger. ${ }^{28} \mathrm{We}$ first investigated the effect of mROS inhibition in mdrladeficient chronic colitis. Here, we tested the effects of colonic MitoQ $(10 \mu \mathrm{M})$, rotenone, and vehicle only in $m d r 1 a$-deficient mice with clinical evidence of chronic colitis (10-12 weeks old, all with chronic diarrhea) (Figure 4a). Colonic MitoQ improved colitis in mdrla-deficient mice (Figure $4 \mathbf{b}-\mathbf{d}$; Supplementary Figure S6a). In contrast to earlier data from mdrla-deficient mice with no colitis, rotenone surprisingly did not further exacerbate chronic colitis. We think this may be due to the intrinsic nature of mdr1a-deficient colitis model where the main driving factor is increased barrier permeability. Hence, colonic mitochondrial dysfunction in this setting acted as a triggering factor and once barrier function was compromised resulting in colitis, rotenone has a lesser role to worsen colitis in this model. Here, the histological severity scores in both rotenone and control groups were 8 and 9.5, respectively, out of the maximal score 12 . The high baseline made it difficult to test for an exacerbating role for rotenone. In acute DSS colitis, colonic MitoQ delayed the onset in mdrladeficient mice (Figure 4f) and significantly attenuated colitis in WT mice (Figure 4g; Supplementary Figure S6b). In both settings, there were histological improvements in the severity of colitis (Figure 4h). We also showed that MitoQ improved clinical recovery following 5 days of DSS challenge (Figure 4I; Supplementary Figure S6c). In this setting, we tested only WT mice, as mdrla-deficient mice were too unwell after DSS induction. Two recent studies corroborate the protective effects of MitoQ in acute DSS colitis (although via oral route). ${ }^{29,30}$ Inhibiting the effects of mROS is therefore beneficial in acute and chronic colitis, as well as during the recovering phase of colitis. 


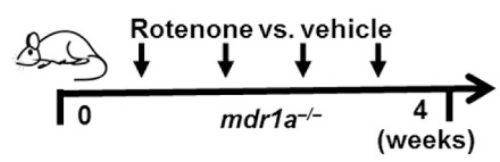

d

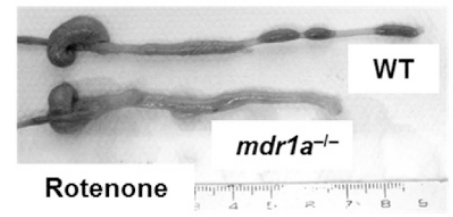

g
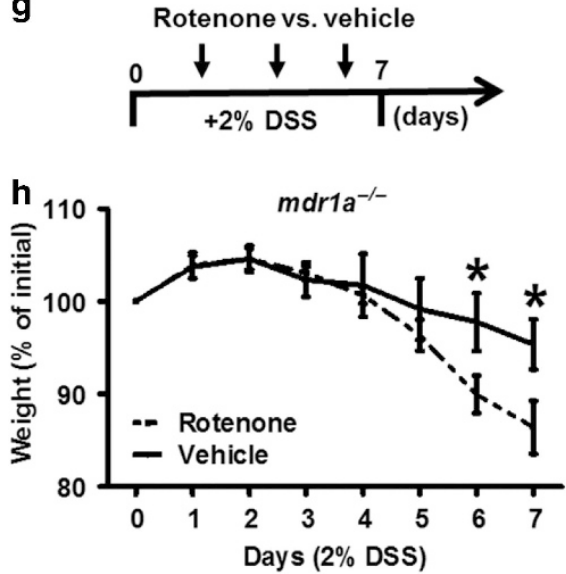

b

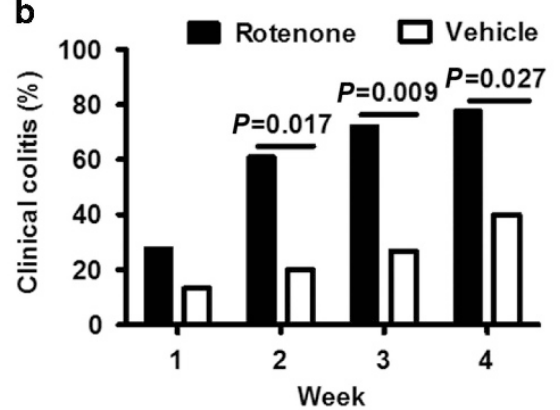

e

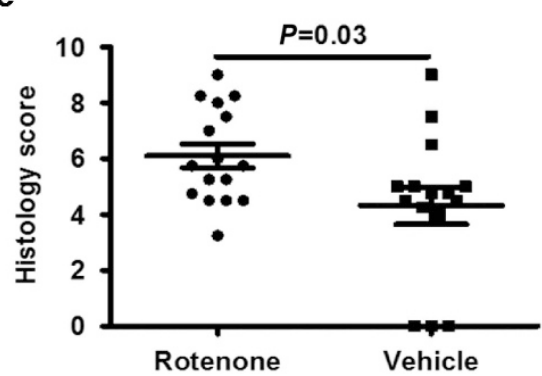

i

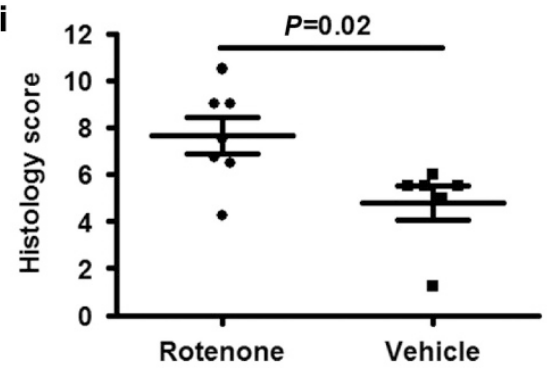

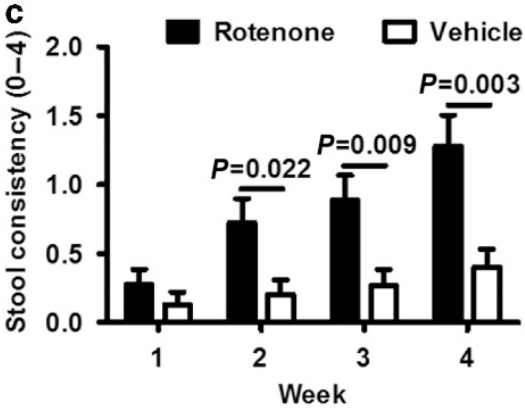

f

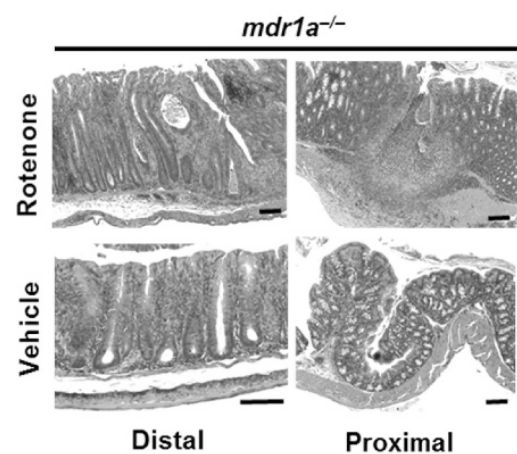

j

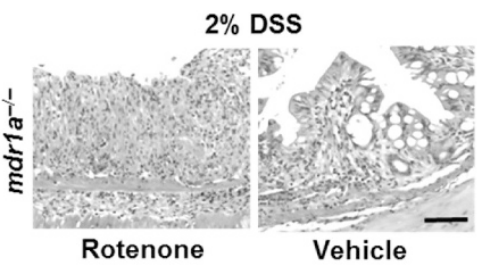

Figure 3 Experimental induction of mROS accelerates spontaneous colitis in mdr1a-deficiency and in acute DSS colitis: (a) Experimental protocol of 4-week colonic administration of rotenone vs. vehicle $\left(3 \times\right.$ per week). (b) Prevalence of clinical colitis in rotenone vs. vehicle-treated $\mathrm{mdr}^{-1 a^{-/-}}$at weekly intervals ( $n=18$ and 15 mice treated with rotenone and vehicle, respectively). (c) Stool consistency measurements in rotenone vs. vehicle treated $\mathrm{mdr1a}^{-1-}$ at weekly intervals. (d) Representative images of $m \mathrm{dr}^{-1 a^{-1-}}$ and WT whole colons treated with colonic rotenone. (e) Histology scores of rotenone vs. vehicle-treated $m d r 1 a^{-1-}$ colons. (f) Representative H\&E distal and proximal colons; and histology scores of rotenone vs. vehicle-treated mdr1a ${ }^{-/-}$, black scale bars $100 \mu \mathrm{m}$. (g) Experimental protocol of colonic rotenone vs. vehicle - three times during 7-day $2 \%$ DSS in drinking water. (h) Daily percentage of change from initial weight in rotenone vs. vehicle-treated $\mathrm{mdr} \mathrm{a}^{-1-}$ acute DSS colitis ( $n=7$ per group). (i,j) Representative histology scores and H\&E colons of rotenone vs. vehicle-treated mdr1a $^{-1-}(n=7$ per group) in acute DSS colitis, black scale bar $50 \mu \mathrm{m}$. All data represent mean \pm s.e.m. DSS, dextran-sulfate sodium colitis; H\&E, hematoxylin and eosin; mdr1, multidrug resistance-1; mROS, mitochondrial reactive oxygen species; WT, wild type. A full color version of this figure is available at the Mucosal Immunology journal online.

\section{MDR1 and SOD2 genes are differentially expressed in human IBD, and intestinal epithelial-specific deletion of SOD2 leads to increased susceptibility to experimental colitis}

To further investigate the role of MDR1 and mROS in human IBD, we performed an in silico analysis of our IBD colonic microarray data set (Gene Expression Omnibus GSE11223 and GSE20881) derived from a cohort of 67 individuals with UC, 53 with CD, 14 non-IBD colitis, and 31 healthy controls. ${ }^{13,31}$ Colonic gene microarray expression data were available from 118 inflamed IBD, 110 non-inflamed IBD, and 50 healthy non-IBD colonic biopsies. In addition to significant downregulation of MDR1 expression in inflamed vs. non-inflamed IBD intestinal biopsies and inflamed IBD vs. healthy non-IBD intestinal biopsies $(P<0.0001$ and 0.0003 , respectively), we interestingly found differential expression of SOD2 expression (upregulated) in both analyses $(P<0.001$ and 0.0002$)$ (Figure 5a and $\mathbf{b}$ ). $M D R 1$ negatively correlated with SOD2 $(r=-0.47 ; P<0.0001)$ in line with our earlier findings in mdrla-deficient mice and shMDR1 CECs (Figure 5c). It is essential to determine whether primary ROS-mediated mitochondria dysfunction acts causally to promote the development of colitis. Furthermore, there may be factors such as variability in tissue penetration and retention that cannot be completely accounted for in the colonic rotenone exposure model. Therefore we genetically deleted the SOD2 gene from the intestinal epithelium by crossing $\mathrm{SOD} 2^{\text {floxedfloxed }}$ mice with mice expressing Cre recombinase under the control of the intestinal epithelial-specific villin promoter to $S O D 2^{I E C-K O}$ mice (Figure 5d). Constitutive deletion of SOD2 gene in mice resulted in death early after birth from 
b

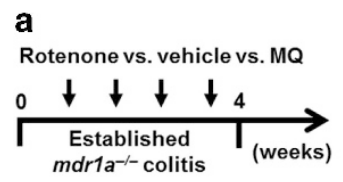

e

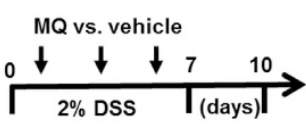

f

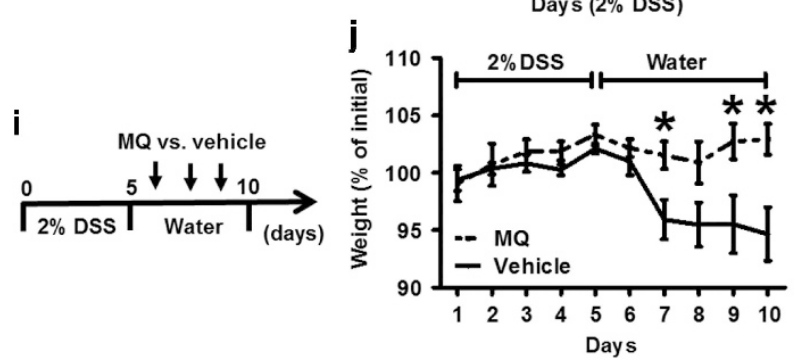

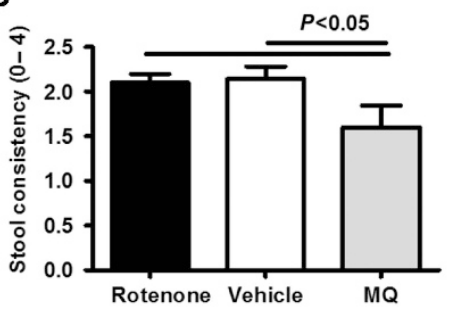

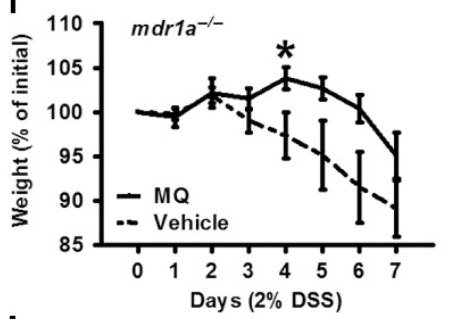

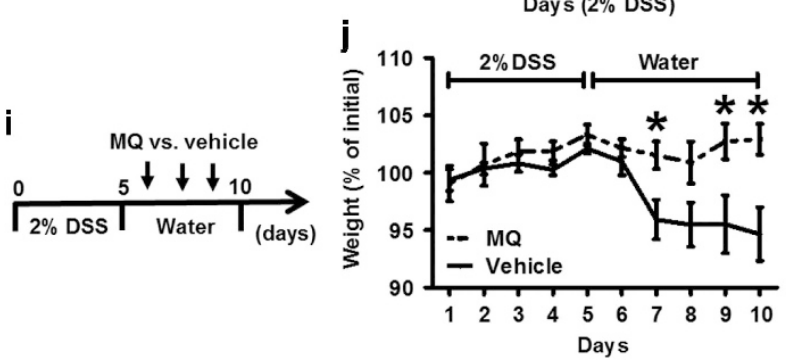

C
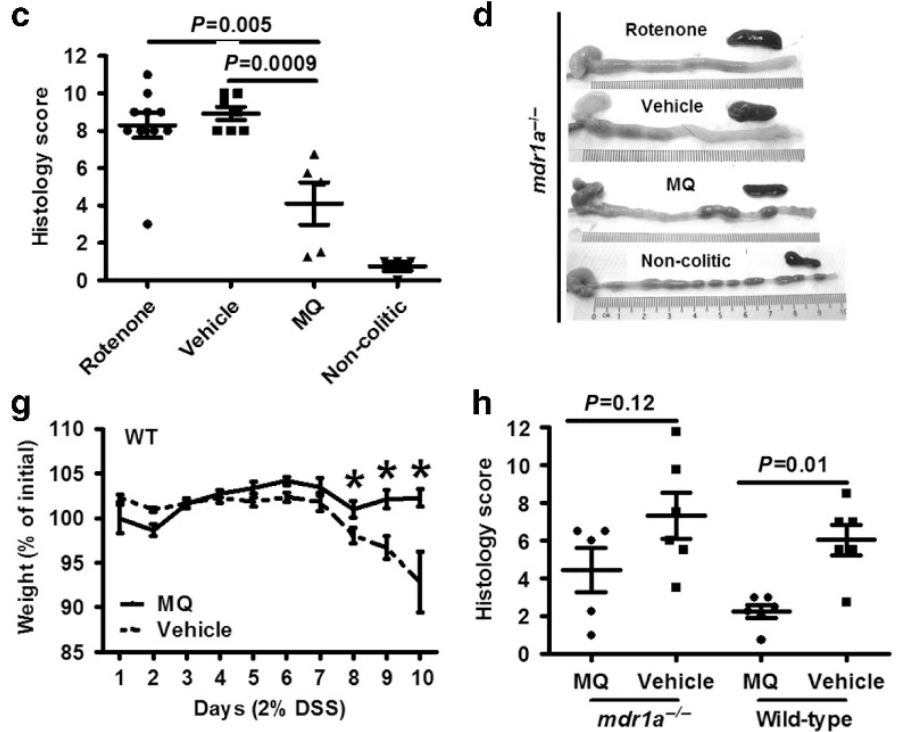

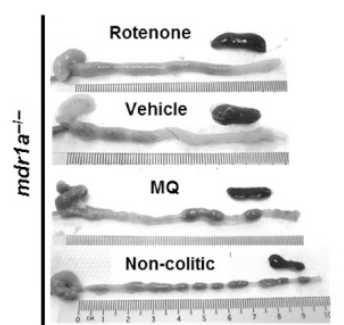

$m d r 1 a^{-/}$

Figure 4 Inhibition of mitochondria ROS effects attenuates and promotes recovery from colitis: (a) Experimental protocol of 4-week colonic administration of rotenone vs. vehicle vs. MQ $\left(3 \times\right.$ per week) in $m d r 1 a^{-/-}$with established chronic colitis. (b) Stool consistency measurements in rotenone vs. vehicle treated vs. MQ in $m d r 1 a^{-/-}$at weekly intervals. (c) Histology colitis scores of rotenone vs. vehicle vs. MQ vs. non-colitic $m d r 1 a^{-/-}$ mice. (d) Representative whole colons and spleen sizes of and H\&E of rotenone vs. vehicle vs. MQ vs. non-colitic mdr1a ${ }^{-/-}$mice. (e) Experimental protocol of colonic MQ vs. vehicle $-3 \times$ administrations during 7-day $2 \%$ DSS colitis protocol. (f, g) Daily percentages change from initial weight clinical colitis in MQ vs. vehicle in $m d r 1 a^{-1-}(n=6$ per group) and WT mice ( $n=6$ per group), respectively. (h) Histology colitis scores in MQ vs. vehicle-treated $\mathrm{mdr} \mathrm{a}^{-/-}$and WT mice, scale bar $50 \mu \mathrm{m}$. (i) Experimental protocol of colonic MQ vs. vehicle treatment after 5 days of $2 \%$ DSS colitis protocol in WT mice. (j) Daily percentages of change from initial weight in MQ vs. vehicle-treated WT, ( $n=8 / 7$, respectively). All data represent mean \pm s.e.m. DSS, dextransulfate sodium colitis; H\&E, hematoxylin and eosin; mdr1, multidrug resistance-1; MQ, MitoQ; ROS, reactive oxygen species; WT, wild type. A full color version of this figure is available at the Mucosal Immunology journal online.

cardiac and liver failure. ${ }^{32}$ In our study, $S O D 2^{I E C-K O}$ mice were born in normal Mendelian ratio and had no overall abnormalities in the morphology of ileum and colon. Although they did not exhibit spontaneous colitis, $S O D 2^{I E C-K O}$ developed more severe colitis following DSS treatment compared to SOD2 $2^{\text {floxed/ }}$ floxed littermates (Figure 5e-g). Our data in $S O D 2^{\mathrm{IEC}-\mathrm{KO}}$ mice provide evidential support for the loss of mitochondrial homeostasis in CECs as a specific factor in determining susceptibility to colitis.

\section{Associations of genes regulating mitochondrial function with human IBD}

Finally, to demonstrate clinical relevance of mitochondrial dysfunction as a new mechanistic factor in IBD, we exploited data from the latest meta-analysis of genome-wide association studies of all European ancestry IBD patients vs. European controls (22,575 CD, 20,417 UC, and 53,536 controls). ${ }^{6}$ The most significant loci in the regions of $A B C B 1$ (encoding MDR1) and SOD2 genes showed associations with $P=3.19 \times 10^{-3}$ and $3.04 \times 10^{-3}$, respectively (below genome-wide significance).
We performed an independent unbiased analysis (using GO term for mitochondria, GO:0005739 using the Mitominer program http://mitominer.mrc-mbu.cam.ac.uk/release-3.1) and identified $29(5.0 \%)$ additional genes involved in mitochondria function within the subset of 574 (out of total 22,353 ) genes within $100 \mathrm{~kb}$ of a genome-wide significant IBD locus from the same meta-analysis data set above (Supplementary Table S1). The top three positional candidate gene associations are: SLC25A28 $\left(P=1.70 \times 10^{-26}\right)$ encoding mitoferrin-2, VARS encoding valine-tRNA ligase $\left(P=4.83 \times 10^{-26}\right)$, and $R N F 5\left(P=9.47 \times 10^{-24}\right)$ encoding E3 ubiquitin-protein ligase RNF5 are involved in mitochondria iron, tRNA transport, and ubiquitination, respectively. ${ }^{33-35}$ Notable also, are the associations of the HSPA1-A, $-B$, and $-L\left(P=1.88 \times 10^{-23}\right)$ genes that encode the heat shock protein 70 , which is integrally involved in the mitochondria unfolded protein responses, crucial for mitochondria protein homeostasis and biogenesis. ${ }^{36}$ This suggests that the mitochondria's contribution is likely more important than previously recognized in IBD. 

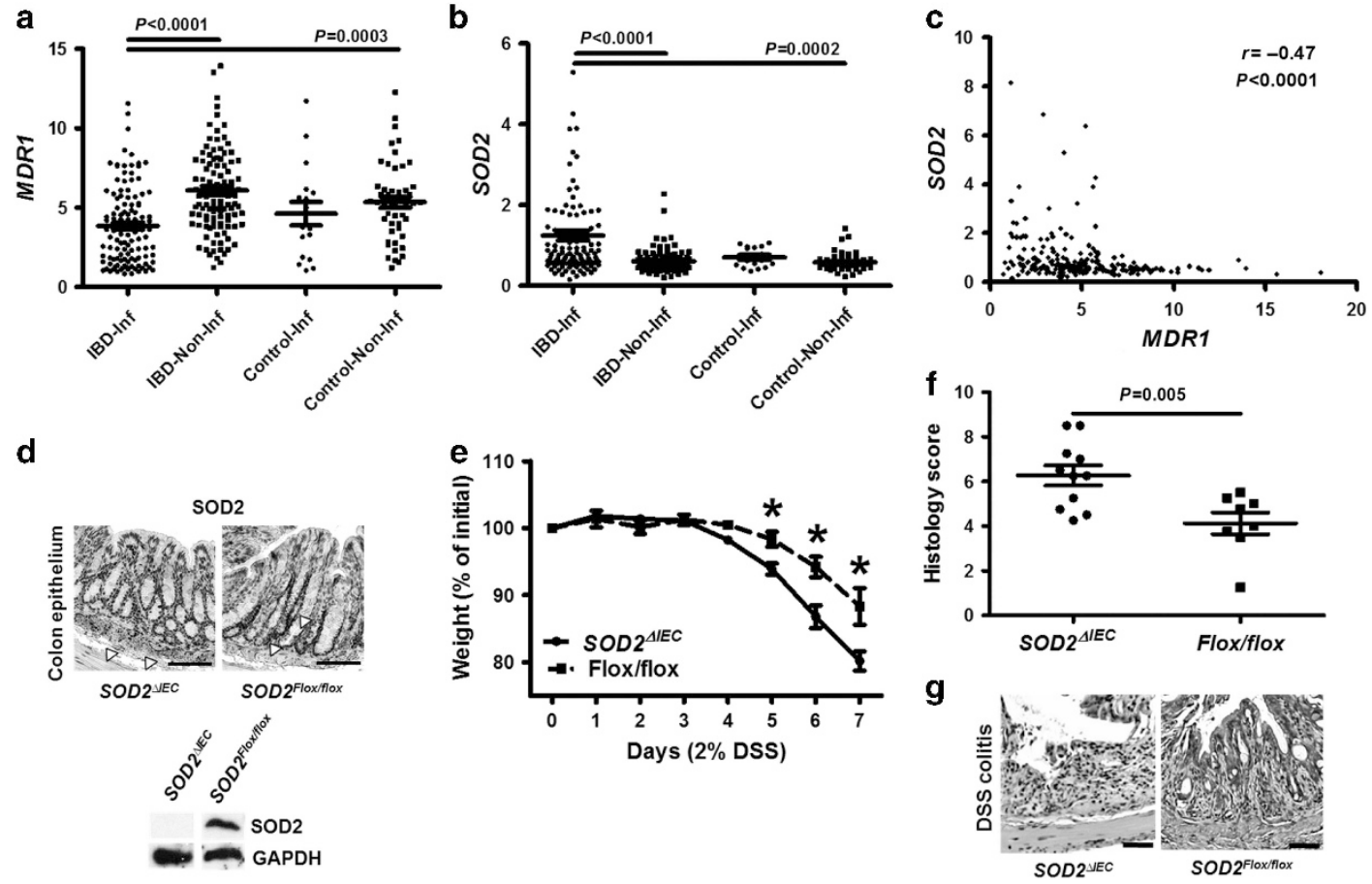

Figure 5 MDR1 and SOD2 genes are differentially expressed in human IBD, and intestinal epithelial-specific deletion of SOD2 leads to increased susceptibility to experimental colitis: (a, b) In silico analysis of MDR1 and SOD2 from 120 IBD patients (67 UC, 53 CD, 17 non-IBD colitis, and 31 healthy controls); (c) Spearman's correlation test of MDR1 and SOD2 expression in 120 IBD patients. (d) Representative immunohistochemistry and Western blotting of SOD2 of colons (a) of SOD2 $2^{I E C-K O}$ and SOD2 $2^{\text {flox fllox }}$ of CECs, black scale bars $100 \mu \mathrm{m}$, yellow arrows showing SOD2 staining. (e) Daily percentages of weight change from initial weight in acute $2 \%$ DSS colitis comparing $S O D 2^{l E C-K O}$ vs. SOD2 flox/flox $(n=12 / 8$, respectively). (f) Histology colitis scores in $S O D 2^{I E C-K O}$ vs. SOD $2^{\text {flox flox }}$ mice $\left(n=12 / 8\right.$, respectively) after $2 \%$ DSS colitis. (g) Representative H\&E staining in SOD2 ${ }^{I E C-K O}$ vs. SOD2 $2^{\text {flox fllox }}$ mice after $2 \%$ DSS colitis, black scale bars $100 \mu \mathrm{m}$. All data represent mean \pm s.e.m. CD, Crohn's disease; CECs, colonic epithelial cells; Control-Inf, non-IBD colitis; Control-Non-Infl, non-inflamed gut biopsies from non-IBD individuals; DSS, dextran-sulfate sodium colitis; H\&E, hematoxylin and eosin; IBD, inflammatory bowel diseases; IBD-Inf, inflamed IBD gut biopsies; IBD-Non-Inf, non-inflamed IBD gut biopsies; MDR1, multidrug resistance-1; SOD2, superoxide dismutase-2; UC, ulcerative colitis. A full color version of this figure is available at the Mucosal Immunology journal online.

\section{DISCUSSION}

In this study, we define a key mitochondrial protective mechanism conferred by MDR1. Here, we present clear evidence of increased mitochondrial damage and consequential dysfunction following MDR1 deficiency with increased susceptibility to colitis driven by mitochondrial dysfunction in vivo. We therefore highlight a cogent model to further support mitochondrial dysfunction as an important mechanistic component in the pathogenesis of IBD (reviewed by Novak and Mollen). ${ }^{37}$ We show conceptually that at the dynamic colonic mucosal interface in particular, mitochondria homeostasis requires tight regulation to prevent the deleterious downstream effects on epithelial integrity, activation of pro-inflammatory responses, and release of mitochondria DAMPs as bona fide inflammatory triggers. The mitochondria participate in a broad range of innate responses to viral, bacterial, and cellular damage. ${ }^{38}$ Recently mitochondria have an increasingly recognized role in inflammation ${ }^{38}$ and are implicated in human diseases with underlying inflammatory pathologies, such as diabetes, ${ }^{39}$ multiple sclerosis, ${ }^{40}$ lung fibrosis, ${ }^{41}$ and cardiovascular disease. ${ }^{42}$

Our data suggest an intricately linked picture, where MDR1 protects against damaging mitochondrial factors arising from the luminal environment via its efflux properties in addition to exerting an influence on key homeostatic responses such as energy production and autophagy. The compositional factors within the lumen in this context; and alternatively, mechanisms that affect the functionality of MDR1 have not yet been studied in detail. As an ATP-dependent efflux transporter, gut energy deficiency is a likely important factor. In the colon, mitochondria rely on the gut microbiota and their production of shortchain fatty acid for energy ${ }^{43}$ and CEC energy deficiency has been previously purported to be an IBD mechanism. Mouse lines with mitochondria genomic variants linked to high ATP production are protected against induced colitis. ${ }^{44}$ While analysis of cellular bioenergetics in primary explants showed impairment in energy production, our in vitro CEC data indicate a more modest effect without the contribution of the luminal flora, with decreased CEC spare respiratory capacity in the presence of diminished MDR1 activity. The lack of baseline $\mathrm{O}_{2}$ consumption rate differences ex vivo and in vitro may reflect fundamental differences in genetic knockdown rather than knockout, inherent bioenergetic properties of colonic "cancer" cell line and in vitro conditions that provided a more stable environment for mitochondrial function. In mouse, colonization of specific gut commensals can influence the level of MDR1 
gene expression. ${ }^{45}$ Hence, it is conceivable that a primary effect of MDR1 deficiency on mitochondrial bioenergetics is potentiated by factors present in the luminal environment in the colon, chiefly the microbiota. In IBD, high levels of mitochondria damage, notably within the electron transport chain have been shown in $\mathrm{CECs}^{46}$ and the resident luminal microbiota are strongly implicated in the pathogenesis of IBD. ${ }^{8}$

The evidence to support directly harmful effect of the microbiota on the mitochondria is noteworthy. Individuals with IBD have higher levels of anerobic sulfate-reducing bacteria, which can produce metabolites that can damage the mitochondria, inhibit cytochrome $c$, and short-chain fatty acid oxidation. ${ }^{47}$ Within the gut flora, several clinically relevant pathogens including enteropathogenic Escherichia coli $^{48}$ Helicobacter pylori, ${ }^{49}$ and Salmonella typhimurium ${ }^{50}$ target effector proteins to the mitochondria. Infection with Citrobacter rodentium has been shown to disrupt mitochondrial function and structure in mice. ${ }^{51}$ These lines of evidence suggest that the gut mitochondria occupy a distinct position, as they are more exposed to damaging luminal factors, more so than in other tissues. Hence, it follows that different protective mechanisms are shaped to counter this challenge. In a proteomic analysis across 14 different mouse tissues, Pagliarini et $a .^{52}$ demonstrated that intestinal epithelial mitochondria have a distinct proteomic profile and notably, have higher expression of $\mathrm{ABC}$ transporters. MDR1 (although part of the $A B C$ superfamily) is not expressed on the mitochondria membrane $e^{53}$ but the closely analogous functions of these transporters highlight the importance of this protective role for the mitochondria in the gut.

Beyond the microbiota, the role of luminal mitochondrial toxins as triggers in IBD is unknown but seems plausible. Findings from PD, a condition characterized by marked mitophagy impairment ${ }^{54,55}$ provides insight into how this might work in IBD pathogenesis. Here an environmental mitochondrial toxin, N-methyl-4-phenyl-1,2,3,6-tetrahydropyridine (MPTP) represents a clear trigger for clinical PD. Like rotenone, $\mathrm{N}$-methyl-4-phenyl-1,2,3,6-tetrahydropyridine is a complex I inhibitor of the electron transport chain that can induce acute PD that is indistinguishable from idiopathic PD. ${ }^{56-58}$ Current literature suggests that rotenone may possess potential effects on the direct aggregation of protein aggregates such as $\alpha$-synuclein, ubiquitin, and GAPDH; alterations of dopamine metabolism; and microglial activation via NFKB activation. ${ }^{26}$ There may be off-target actions too, but given its dominant effect on mitochondria respiratory function, these may be difficult to detect. Even though the role for autophagy in general is clearly established in IBD, mitophagy has not yet been fully studied in this context. ${ }^{5,59}$ Strikingly and on further analysis however, genes involved in mitophagy (PARK7 chr1p36; $P=4.8-10^{-9}$ for UC; and LRRK2 chr12q12; $P=6.2$ $\times 10^{-21}$ for $\mathrm{CD}$ ) are strongly associated with IBD in genomewide association studies. In parallel, defective mitophagy has been demonstrated in CECs derived from Irgm1-deficient mice, implicating this pathway in another IBD-associated gene polymorphism. ${ }^{23}$ Like the virus-plus-susceptibility gene interaction that determines $\mathrm{CD}$ susceptibility as proposed by Cadwell et al. ${ }^{60}$ in 2010, we put forward the concept of mitochondrial toxin-plus-genetic susceptibility. In this regard, we show that rotenone can accelerate the development of spontaneous colitis in the susceptible mdrla-deficient model and in acute-induced DSS colitis, a setting when a second "hit" along with rotenone exacerbates the severity of colitis. We believe that this is the first description of such kind although the protective effects of mitochondrial-specific antioxidant (MitoQ) and more generally, antioxidants such as ascorbic acid have been described in experimental colitis. ${ }^{29,30,61}$

Mitochondrial dysfunction via ROS production is a major mediator of inflammation and can directly modulate the NLRP3 inflammasome, NFKB, and MAPK signaling pathways. $^{62,63}$ Mitochondrial ROS also promote the cytosolic release of mitochondrial DNA, which mediates NLRP3 inflammasome activation and can directly trigger TLR9 signaling on neutrophils. ${ }^{64-66}$ Accumulation of dysfunctional mitochondria perpetuate the production of mROS, amplifying innate inflammatory responses such as type I interferon ${ }^{67}$ and endotoxin-induced interleukin-1 $\beta$ production. ${ }^{68}$ Mitochondrial ROS inhibits autophagy, the major process for removal of damaged mitochondria. ${ }^{64}$ Defective autophagy in turn allows for the escape of pro-inflammatory mitochondrial DAMPs. ${ }^{65,66}$ Our published colonic microarray study showing that gene sets regulating the mitochondria $\left(P=4.7 \times 10^{- \text {he }}\right)$ and oxidoreductase functions $\left(P=1.4 \times 10^{-\mathrm{a}}\right)$ were most differentially expressed comparing distal (where UC invariably affects) vs. proximal colon in UC. Further in silico analyses showed a negative correlation between $M D R 1$ and SOD2, and our data showing increased susceptibility to DSS colitis following intestinal epithelial-specific deletion of the SOD2 gene strongly supports the causal importance of ROS detoxification.

IBD pathogenesis is complex and encompasses multiple factors, which may trigger disease onset, heighten susceptibility, potentiate severity, and/or promulgate non-resolution of inflammation. These factors combine to shape the many phenotypes of IBD. In this context, our data importantly highlight the mitochondria's contribution as a new "jigsaw" piece, a hitherto underexplored focus within this complex framework of factors. Specific pharmacological MDR1 augmentation is a translational challenge due to induction of drug resistance as one known problem. However, our studies open up a new landscape of potential therapeutic approaches targeting downstream effects of mROS-DAMP, cellular bioenergetics, and mitophagy as clear examples. Furthermore, we show that $5 \%$ of genes highlighted by IBD genome-wide association studies are functionally linked to the maintenance of mitochondria health. Hence, the focus on mitochondrial dysfunction has broad translational applications, which will lead to many new mechanistic perspectives and therapeutic opportunities in IBD.

\section{METHODS}

Mouse. The FVB/129 $\mathrm{mdrla}^{-/-}$and $\mathrm{WT}$ mouse lines were purchased from Taconic (Hudson, NY). C57/BL6 mice carrying floxed 
allele flanking SOD2 gene was provided by Dr Shimizu, Chiba University, Japan and re-derived in University of Edinburgh. Villin-Cre recombinase C57/BL6 mice were purchased from Jackson laboratory. All colonies were maintained in specific pathogen-free conditions (specifically Helicobacter-free) in University of Edinburgh. Il10-deficient mice were maintained at the University of North Carolina, Chapel Hill.

Electron microscopy. Mouse or human colonic samples were flushed with sterile phosphate buffered saline and immediately transferred into $3 \%$ electron microscopy grade glutaraldehyde solution in $0.1 \mathrm{M}$ sodium cacodylate buffer, $\mathrm{pH} 7.3$, for $2 \mathrm{~h}$ before further processing. For quantification of abnormal mitochondria, viable cells with five or more abnormal mitochondria were classified as "abnormal". A percentage against total number of IECs (minimum of 100 IECs in $>5$ images) was calculated per mouse.

Mitochondria studies. Mitochondria from CECs or mouse tissues were performed using Dounce homogenizers (30-40 strokes in two different gradients of homogenizers) extracted using Mitochondria Isolation Kit (Sigma-Aldrich, Gillingham, Dorset, UK, MITOISO1). For JC-1 assay, the mitochondria pellets were re-suspended in $100 \mu \mathrm{l}$ storage buffer $+1900 \mu \mathrm{l} \mathrm{JC}-1$ assay buffer + JC-1. Mitochondria suspensions were placed in clear-bottom black 96-well plates: untreated, antimycin $10 \mu \mathrm{g} \mathrm{ml}^{-1}$, rotenone $10 \mu \mathrm{M}$, and CCCP $10 \mu \mathrm{M}$. JC-1 fluorescence was measured using the plate reader at $590 \mathrm{~nm}$. The rate of mitochondria membrane depolarization was measured as the loss of JC-1 fluorescence after $1 \mathrm{~h}$. Measurements were normalized to the untreated group. For mROS studies, T84 CECs were plated and grown in 96-well tissue culture plates for at least $24-48 \mathrm{~h}$ and to $\sim 70 \%$ confluence. Cells were cultured in MitoSOX $(2.5 \mu \mathrm{M})$ or mitotracker Green (100 nM) for $30 \mathrm{~min}$ and then washed in pre-warmed phosphate buffered saline before further culture with antimycin $\left(20 \mu \mathrm{g} \mathrm{ml}^{-1}\right)$, rotenone $(10 \mu \mathrm{M})$, or CCCP $(10 \mu \mathrm{M})$. Relative fluorescence was quantified using the plate reader at Ex/Em 510/580 and 490/516 for MitoSOX and Mitotracker Green, respectively. MitoSOX/MitoGreen relative fluorescence readings were normalized to untreated MitoSOX/ Mitotracker Green cells only.

Cellular energetics studies. Colon and ileum were harvested and placed in DMEM/F12 medium. A $3 \times 3 \mathrm{~mm}$ intestinal section was cut and mounted in Seahorse XF24 culture plate with the apical side of the lumen orientated upwards and maintained in position by an Islet Microcapture screen. The explants were washed twice with Seahorse Assay Media, supplemented with $1 \mathrm{~mm}$ pyruvate and $10 \mathrm{~mm}$ glucose $\left(\mathrm{pH} 7.4\right.$ at $\left.37^{\circ} \mathrm{C}\right)$. These explants were placed into Seahorse Analyzer within $1 \mathrm{~h}$ of tissue harvest. We measured $\mathrm{O}_{2}$ consumption rate following the addition of antimycin/rotenone; providing mitochondrial function. The raw data were normalized to protein content using the Bradford quantification. T84 shMDR1 and shCtrl CECs were plated on Seahorse XF24 cell culture plates. For the mitochondria stress test oligomycin was added added at $2 \mu \mathrm{M}$, followed by $1 \mu \mathrm{M}$ carbonyl cyanide-4-(trifluoromethoxy)phenylhydrazone then antimycin and rotenone $(1 \mu \mathrm{M}$ each). Data were normalized to protein content measured by sulforhodamine B staining (Sigma S1402) to adjust for cell density.

Induction and histological grading of colitis. Colonic rotenone or vehicle were administered using a flexible rectal tube following brief general anesthesia with isofluorane. A volume of $500 \mu \mathrm{l}$ was used in a head-down position for at least $2 \mathrm{~min}$ before removal of anesthesia and then immediately check for any complications. The rotenone concentration of $100 \mu \mathrm{M}$ (volume of $500 \mu \mathrm{l}$ ) equates to $\sim 1 \mathrm{mg} \mathrm{kg}^{-1}$. In prior optimization, we have tested that this volume completely filled the mouse colon to provide total exposure. Acute colitis was induced by 2-3\% DSS (MP Biomedicals, Santa Ana, CA) in drinking water ad libitum for 7-10 days. Mice were monitored daily for weights, presence of diarrhea and blood. In chronic rotenone treatment, mice were assessed at the end of every week. Specifically, we quantified stool consistency: Well-formed/normal $=0$, pasty/semi-formed $=1$, pasty $=2$, diarrhea that does not adhere to anus $=3$, diarrhea that adheres to anus $=4$. For histology, each colonic sample was graded semi-quantitatively (scores $0-3$ per component): (A) Degree of epithelial hyperplasia and goblet cell depletion. (B) Leukocyte infiltration in the lamina propria. (C) Area of tissue affected. (D) Presence of markers of severe inflammation (such as crypt abscesses, submucosal inflammation, and the presence of ulcers). For colitis scores, the mean of proximal and distal colon scores was calculated.

Quantification of mitochondrial DNA. Plasma samples were obtained by cardiac puncture following $\mathrm{CO}_{2}$ killing. Following sampling, blood samples were spun at $5,000 \mathrm{~g}$ for $10 \mathrm{~min}$ at $4{ }^{\circ} \mathrm{C}$. DNA was extracted using Qiagen DNA Blood Mini Kit. Primer sequences for cytochrome $c$ oxidase sub-unit I (mCOI, forward 5-GCCCCAGATATAG-CATT CCC-3; Reverse, 5-GTTCATCCTGTTCCTGCTCC-3) with quantitative PCR performed using $2 \times$ SYBR Green Fast mix (Applied Biosystems, Foster City, CA). quantitative PCR reactions were conducted in an ABI7900 Fast Real-Time PCR System (Applied Biosystems) with the following thermal profile: 1 cycle $95^{\circ} \mathrm{C}$ for $20 \mathrm{~s}$; 40 cycles of $95^{\circ} \mathrm{C}$ for $3 \mathrm{~s}$, and $60^{\circ} \mathrm{C}$ for $30 \mathrm{~s}$. Mitochondrial DNA levels were quantified based on the following calculation: $c=Q \times V_{\mathrm{DNA}} /$ $V_{\mathrm{PCR}} \times 1 / V_{\text {ext; }}$ where $c$ is the concentration of DNA in plasma (copies per $\mu \mathrm{l}$ ); $Q$ is the quantity (number of copies) of DNA determined by the quantitative PCR system; $V_{\mathrm{DNA}}$ is the total volume of eluted plasma DNA solution obtained after extraction $(40 \mu \mathrm{l}) ; V_{\mathrm{PCR}}$ is the volume of plasma DNA used for PCR $(40 \mu \mathrm{l})$; and $V_{\text {ext }}$ is the volume of plasma extracted $(200 \mu \mathrm{l})$. Quantification was achieved relative to a known concentration of PCR amplicon obtained from DNA extracted from mouse CEC mitochondria using the method previously reported.

Microarray data set and analysis. Full details of tissue acquisition and process are previously detailed. ${ }^{13}$ The whole data set is available at Gene Expression Omnibus (http://www.ncbi.nlm.nih.gov/geo/ (accessed 18 July 2008) $^{6}$ ) accession: GSE11223 and GSE20881.

Genetic analyses. The presented data were derived from the metaanalysis of all European ancestry IBD patients vs. European controls. ${ }^{6}$ Analysis was done in R 3.2.2 (R Foundation for Statistical Computing, Vienna, Austria) using the Genomic Ranges package. ${ }^{33}$ The UCSC hg19 Known Gene Transcripts were filtered to keep only those with an associated Entrez Gene ID. For each Gene ID the earliest start and latest end position was taken from all of the transcripts for that gene. All $P$-values within $100 \mathrm{~kb}$ of each range were then associated with the respective Gene ID.

shRNA knockdown studies and transfection. T84 cells (purchased from ATCC) cells expressing MDR1 were transfected with MDR1 and control shRNA plasmids (SABiosciences, Qiagen, Germantown, MD) and selected using G418 $\left(600 \mu \mathrm{g} \mathrm{ml}^{-1}\right)$. Cells were cultured in $\mathrm{DMEM} / \mathrm{F} 12$ medium with $10 \%$ fetal calf serum, $100 \mathrm{U} \mathrm{ml}^{-1}$ penicillin, and $100 \mu \mathrm{g} \mathrm{ml}^{-1}$ streptomycin. GFP-LC3 plasmid was transfected by Lipofectamine method (1:2 ratio) with GFP-plasmid controls to confirm transfection efficiency. Transfection was carried out on shMDR1 and shCtrl CECs grown on glass coverslips, which were then fixed with $4 \%$ paraformaldehyde and then viewed under fluorescence microscope.

Colonic epithelial cell isolation. Colons were removed and immediately placed in cold sterile phosphate buffered saline. Colons were opened longitudinally and further washed with buffer (154 mM $\mathrm{NaCl}+1 \mathrm{~mm}$ dithiothreitol, DTT) three times. These were then placed in $5 \mathrm{ml}$ dissociation buffer $\left(130 \mathrm{~mm} \mathrm{NaCl}, 1 \mathrm{~mm} \mathrm{Na} \mathrm{N}_{2}\right.$ EDTA, $10 \mathrm{~mm}$ HEPES, $10 \%$ FCS, 1 mM DTT) in a bijou and placed on a shaker at 140 r.p.m. at $37^{\circ} \mathrm{C}$ for $15 \mathrm{~min}$. Dissociated cells were placed in $15 \mathrm{ml}$ Falcon tube on ice. Incubation in dissociation buffer was repeated with another $2 \times 5 \mathrm{ml}$. Dissociated cells were spun at 2,500 rpm for $10 \mathrm{~min}$ at $4{ }^{\circ} \mathrm{C}$. Pellet was washed with phosphate buffered saline, spun at $5,000 \mathrm{rpm}$ for $5 \mathrm{~min}$ at $4^{\circ} \mathrm{C}$. 
SUPPLEMENTARY MATERIAL is linked to the online version of the paper at http://www.nature.com/mi

\section{ACKNOWLEDGMENTS}

This work was supported by MRC grant G0701898, Crohn's and Colitis UK M16-1, ECCO IBD Investigator's Award 2010, Chief Scientist Office ETM/ 75 awarded to GTH; NIH grants P01 DK 094779 and R01 DK053347 awarded to RBS; Wellcome Trust Investigator Award WT100981MA to NMM; Wellcome Trust grant WT096497 to DAD and AGR; Wellcome Trust WT097943 to NAK; Wellcome Trust Biomedical Vacation Scholarship 202597/Z/16/Z to ETSC; EU FP-7 grants 305676-2 and 305479-2 to JS.

\section{AUTHOR CONTRIBUTION}

G.T.H. planned and carried out experiments, analyzed data, and wrote the manuscript. R.E.A. performed the in vitro cell line work, transfection studies, and gene/protein expression studies. R.N.C., E.T.S.C., R.E.A., and NM planned, carried out, and analyzed the mitochondria Seahorse experiments. B.L. planned and assisted in mouse colitis studies, performed histological analysis, and TEM work. D.A.D. set up the mitochondria D.N.A. quantitative P.C.R. work and performed data analysis. N.A.K. recruited I.B.D. subjects and prepared plasma samples for human mitochondrial D.N.A. work, and performed additional in silico microarray and genetic analyses. C.L.N. carried out original work of human IBD microarray work. T.S. generated SOD2 mouse mutants. R.B.S. provided C57/BL6 transgenic strains ( $\mathrm{mdr} 1 \mathrm{a}^{-1-}$ and $\mathrm{i} / 10^{-/-}$). R.B.S., A.G.R., J.P.I., and J.S. provided intellectual input into planning of experiments, data analysis and contributed to the writing of the manuscript. All authors reviewed and approved the final manuscript.

\section{DISCLOSURE}

The authors declared no conflict of interest.

(c) 2018 Society for Mucosal Immunology

\section{REFERENCES}

1. Danese, S. \& Fiocchi, C. Ulcerative colitis. N Engl J Medicine 365, 1713-1725 (2011).

2. Boyapati, R., Satsangi, J. \& Ho, G.T. Pathogenesis of Crohn's disease. F1000Prime Rep 7, 44 (2015).

3. Molodecky, N.A. et al. Increasing incidence and prevalence of the inflammatory bowel diseases with time, based on systematic review. Gastroenterology 142, 46-54, e42, quiz e30 (2012).

4. Ho, G.-T., Boyapati, R. \& Satsangi, J. Ulcerative colitis. Medicine 43, 276-281 (2015).

5. Jostins, L. et al. Host-microbe interactions have shaped the genetic architecture of inflammatory bowel disease. Nature 491, 119-124 (2012).

6. Liu, J.Z. et al. Association analyses identify 38 susceptibility loci for inflammatory bowel disease and highlight shared genetic risk across populations. Nat Genet 47, 979-986 (2015).

7. McCole, D.F. IBD candidate genes and intestinal barrier regulation. Inflamm Bowel Dis 20, 1829-1849 (2014).

8. Sartor, R.B. \& Wu, G.D. Roles for intestinal bacteria, viruses, and fungi in pathogenesis of inflammatory bowel diseases and therapeutic approaches. Gastroenterology 152, 327-339.e4 (2016).

9. Dean, M., Rzhetsky, A. \& Allikmets, R. The human ATP-binding cassette (ABC) transporter superfamily. Genome Res 11, 1156-1166 (2001).

10. Ambudkar, S.V. et al. Biochemical, cellular, and pharmacological aspects of the multidrug transporter. Annu Rev Pharmacol Toxicol 39, 361-398 (1999).

11. Thiebaut, F. et al. Cellular localization of the multidrug-resistance gene product P-glycoprotein in normal human tissues. Proc Natl Acad Sci USA 84, 7735-7738 (1987).

12. Panwala, C.M., Jones, J.C. \& Viney, J.L. A novel model of inflammatory bowel disease: mice deficient for the multiple drug resistance gene, mdr1a, spontaneously develop colitis. J Immunol 161, 5733-5744 (1998).

13. Noble, C.L. et al. Regional variation in gene expression in the healthy colon is dysregulated in ulcerative colitis. Gut 57, 1398-1405 (2008).
14. Langmann, T. et al. Loss of detoxification in inflammatory bowel disease: dysregulation of pregnane X receptor target genes. Gastroenterology 127, 26-40 (2004).

15. Onnie, C.M. et al. Associations of allelic variants of the multidrug resistance gene (ABCB1 or MDR1) and inflammatory bowel disease and their effects on disease behavior: a case-control and meta-analysis study. Inflamm Bowel Dis 12, 263-271 (2006).

16. Resta-Lenert, S., Smitham, J. \& Barrett, K.E. Epithelial dysfunction associated with the development of colitis in conventionally housed mdr1a-/- mice. Am J Physiol Gastrointest Liver Physiol 289, G153-G162 (2005).

17. Kim, S.C. et al. Variable phenotypes of enterocolitis in interleukin 10-deficient mice monoassociated with two different commensal bacteria. Gastroenterology 128, 891-906 (2005).

18. Pankiv, S. et al. p62/SQSTM1 binds directly to Atg8/LC3 to facilitate degradation of ubiquitinated protein aggregates by autophagy. $J$ Biol Chem 282, 24131-24145 (2007).

19. Youle, R.J. \& Narendra, D.P. Mechanisms of mitophagy. Nat Rev Mol Cell Biol 12, 9-14 (2011).

20. Choi, S.W., Gerencser, A.A. \& Nicholls, D.G. Bioenergetic analysis of isolated cerebrocortical nerve terminals on a microgram scale: spare respiratory capacity and stochastic mitochondrial failure. J Neurochem 109, 1179-1191 (2009).

21. Brookes, P.S., Yoon, Y., Robotham, J.L., Anders, M.W. \& Sheu, S.S. Calcium, ATP, and ROS: a mitochondrial love-hate triangle. Am J Physiol Cell Physiol 287, C817-C833 (2004).

22. Adolph, T.E. et al. Paneth cells as a site of origin for intestinal inflammation. Nature 503, 272-276 (2013).

23. Liu, B. et al. Irgm1-deficient mice exhibit Paneth cell abnormalities and increased susceptibility to acute intestinal inflammation. Am J Physiol Gastrointest Liver Physiol 305, G573-G584 (2013).

24. Kaser, A. et al. XBP1 links ER stress to intestinal inflammation and confers genetic risk for human inflammatory bowel disease. Cell 134, 743-756 (2008).

25. Elinav, E. et al. NLRP6 inflammasome regulates colonic microbial ecology and risk for colitis. Cell 145, 745-757 (2011).

26. Xiong, N. et al. Mitochondrial complex I inhibitor rotenone-induced toxicity and its potential mechanisms in Parkinson's disease models. Crit Rev Toxicol 42, 613-632 (2012).

27. Johnson, M.E. \& Bobrovskaya, L. An update on the rotenone models of Parkinson's disease: their ability to reproduce the features of clinical disease and model gene-environment interactions. Neurotoxicology 46, 101-116 (2015).

28. Murphy, M.P. \& Smith, R.A. Targeting antioxidants to mitochondria by conjugation to lipophilic cations. Annu Rev Pharmacol Toxicol 47, 629-656 (2007).

29. Wang, A. et al. Targeting mitochondria-derived reactive oxygen species to reduce epithelial barrier dysfunction and colitis. Am J Pathol 184, 2516-2527 (2014).

30. Dashdorj, A. et al. Mitochondria-targeted antioxidant MitoQ ameliorates experimental mouse colitis by suppressing NLRP3 inflammasomemediated inflammatory cytokines. BMC Med 11, 178 (2013).

31. Noble, C.L. et al. Characterization of intestinal gene expression profiles in Crohn's disease by genome-wide microarray analysis. Inflamm Bowel Dis 16, 1717-1728 (2010).

32. Li, Y. et al. Dilated cardiomyopathy and neonatal lethality in mutant mice lacking manganese superoxide dismutase. Nat Genet 11, 376-381 (1995).

33. Lawrence, M. et al. Software for computing and annotating genomic ranges. PLoS Comput Biol 9, e1003118 (2013).

34. Zhong, B. et al. The ubiquitin ligase RNF5 regulates antiviral responses by mediating degradation of the adaptor protein MITA. Immunity 30, 397-407 (2009).

35. Ryan, M.T. \& Hoogenraad, N.J. Mitochondrial-nuclear communications. Annu Rev Biochem 76, 701-722 (2007).

36. Rath, E. \& Haller, D. Mitochondria at the interface between danger signaling and metabolism: role of unfolded protein responses in chronic inflammation. Inflamm Bowel Dis 18, 1364-1377 (2012).

37. Novak, E.A. \& Mollen, K.P. Mitochondrial dysfunction in inflammatory bowel disease. Front Cell Dev Biol 3, 62 (2015). 
38. West, A.P., Shadel, G.S. \& Ghosh, S. Mitochondria in innate immune responses. Nat Rev Immunol 11, 389-402 (2011).

39. Szendroedi, J., Phielix, E. \& Roden, M. The role of mitochondria in insulin resistance and type 2 diabetes mellitus. Nat Rev Endocrino/ 8, 92-103 (2012).

40. Witte, M.E., Mahad, D.J., Lassmann, H. \& van Horssen, J. Mitochondrial dysfunction contributes to neurodegeneration in multiple sclerosis. Trends Mol Med 20, 179-187 (2014).

41. Bueno, M. et al. PINK1 deficiency impairs mitochondrial homeostasis and promotes lung fibrosis. J Clin Invest 125, 521-538 (2015).

42. Nisoli, E., Clementi, E., Carruba, M.O. \& Moncada, S. Defective mitochondrial biogenesis: a hallmark of the high cardiovascular risk in the metabolic syndrome?. Circ Res 100, 795-806 (2007).

43. Donohoe, D.R. et al. The microbiome and butyrate regulate energy metabolism and autophagy in the mammalian colon. Cell Metab 13, 517-526 (2011).

44. Bar, F. et al. Mitochondrial gene polymorphisms that protect mice from colitis. Gastroenterology 145, 1055-1063, e1053 (2013).

45. Hooper, L.V. et al. Molecular analysis of commensal host-microbial relationships in the intestine. Science 291, 881-884 (2001).

46. Santhanam, S. et al. Mitochondrial electron transport chain complex dysfunction in the colonic mucosa in ulcerative colitis. Inflamm Bowel Dis 18, 2158-2168 (2012).

47. Szabo, C. Hydrogen sulphide and its therapeutic potential. Nat Rev Drug Discov 6, 917-935 (2007).

48. Nagai, T., Abe, A. \& Sasakawa, C. Targeting of enteropathogenic Escherichia coli EspF to host mitochondria is essential for bacterial pathogenesis: critical role of the 16th leucine residue in EspF. J Biol Chem 280, 2998-3011 (2005).

49. Ashktorab, H. et al. Bax translocation and mitochondrial fragmentation induced by Helicobacter pylori. Gut 53, 805-813 (2004).

50. Hernandez, L.D., Pypaert, M., Flavell, R.A. \& Galan, J.E. A Salmonella protein causes macrophage cell death by inducing autophagy. J Cell Biol 163, 1123-1131 (2003).

51. Ma, C. et al. Citrobacter rodentium infection causes both mitochondrial dysfunction and intestinal epithelial barrier disruption in vivo: role of mitochondrial associated protein (Map). Cell Microbiol 8, 1669-1686 (2006).

52. Pagliarini, D.J. et al. A mitochondrial protein compendium elucidates complex I disease biology. Cell 134, 112-123 (2008).
53. Paterson, J.K. \& Gottesman, M.M. P-Glycoprotein is not present in mitochondrial membranes. Exp Cell Res 313, 3100-3105 (2007).

54. Smith, W.W. et al. Kinase activity of mutant LRRK2 mediates neuronal toxicity. Nat Neurosci 9, 1231-1233 (2006).

55. Bonifati, V. et al. Mutations in the DJ-1 gene associated with autosomal recessive early-onset parkinsonism. Science 299, 256-259 (2003).

56. Langston, J.W., Ballard, P., Tetrud, J.W. \& Irwin, I. Chronic Parkinsonism in humans due to a product of meperidine-analog synthesis. Science $\mathbf{2 1 9}$, 979-980 (1983).

57. Betarbet, R. et al. Chronic systemic pesticide exposure reproduces features of Parkinson's disease. Nat Neurosci 3, 1301-1306 (2000).

58. Panov, A. et al. Rotenone model of Parkinson disease: multiple brain mitochondria dysfunctions after short term systemic rotenone intoxication. J Biol Chem 280, 42026-42035 (2005).

59. Franke, A. et al. Genome-wide meta-analysis increases to 71 the number of confirmed Crohn's disease susceptibility loci. Nat Genet 42, 1118-1125 (2010).

60. Cadwell, K. et al. Virus-plus-susceptibility gene interaction determines Crohn's disease gene Atg16L1 phenotypes in intestine. Cell 141, 1135-1145 (2010).

61. Yan, H., Wang, H., Zhang, X., Li, X. \& Yu, J. Ascorbic acid ameliorates oxidative stress and inflammation in dextran sulfate sodium-induced ulcerative colitis in mice. Int J Clin Exp Med 8, 20245-20253 (2015).

62. Bulua, A.C. et al. Mitochondrial reactive oxygen species promote production of proinflammatory cytokines and are elevated in TNFR1associated periodic syndrome (TRAPS). J Exp Med 208, 519-533 (2011).

63. Zhou, R., Yazdi, A.S., Menu, P. \& Tschopp, J. A role for mitochondria in NLRP3 inflammasome activation. Nature 469, 221-225 (2011).

64. Nakahira, K. et al. Autophagy proteins regulate innate immune responses by inhibiting the release of mitochondrial DNA mediated by the NALP3 inflammasome. Nat Immunol 12, 222-230 (2011).

65. Zhang, Q. et al. Circulating mitochondrial DAMPs cause inflammatory responses to injury. Nature 464, 104-107 (2010).

66. Oka, T. et al. Mitochondrial DNA that escapes from autophagy causes inflammation and heart failure. Nature 485, 251-255 (2012).

67. Tal, M.C. et al. Absence of autophagy results in reactive oxygen speciesdependent amplification of RLR signaling. Proc Natl Acad Sci USA 106, 2770-2775 (2009).

68. Saitoh, T. et al. Loss of the autophagy protein Atg16L1 enhances endotoxin-induced IL-1 beta production. Nature 456, 264-268 (2008). 